Dermatologica, 1959;119:383-384

\title{
Register rerum ad vol. 119
}

Originalarbeiten ohne Bezeichnung Original articles without asterisk Travaux originaux sans designation

Demonstrationen * Demonstrations * Demonstrations *

Sammelreferate $* *$

Surveys **

Revues Générales **

Buchbesprechungen *** Book Reviews *** Livres Nouveaux ***

Allergic Encephalomyelitis and "Auto-Allergic" Diseases 258 ***

Allergy and Immunology, Current Problems in $259 * * *$

Antibiotica et Chemotherapia, Vol. $6258 * * *$

Besnier-Boeck-Schaumann, Morbus (M.

B.) $240 * *$ Brocq Morbus: Kollodiumbabys 164

Cancers de la peau, modifications cuta-

nées consécutives à Tirradiation de 221 Carcinoma, Multiple Superficial Basal

Cell, Treated with Demecolcine 20 Cheilitis granulomatosa $247 * *$ Chemotherapia et

Antibiotica, Vol. 6

$258 * * *$ Cignolin, Toxizität und Stoffwechsel des

211

Darier-Roussy's Sarcoid 300

Dermatologie, aktuelle Probleme der $195 * * *$

Dermatologie, Atlas der $381 * * *$

Dermatologie und Venerologie, Bd. III/1 $260 * * *$

Dermatologie und Venerologie, Bd. IП/2 $381 * * *$

Désensibilisation dans $\Gamma$ eczéma experimental du cobaye 186

Disulfirampsychose bei der Psoriasis-Behandlung 238

Drugs, Sensitivity Reactions to $259 * * *$

Eczema experimental du cobaye, désensibilisation dans $\Gamma 186$

Eczema professionnel au nickel, quel-ques remarques sur $\Gamma 40$

Ekzem, experimentelles, durch Kontakt mit Dinitrochlorbenzol an Ratten und Kaninchen 294

Encephalomyelitis, experimental allergic, and the "Auto-Allergic" Diseases $258 * * *$

Enzymes $364 * *$

Erythema elevatum et diutinum 65

Gefäßerkrankungen, Bewertung der Rie-senzellen bei, und Periarteriitis nodosa 1

Granuloma annulare $250 * *$

Granulomatosis disciformis chronica et progressiva $254 * *$

Granulom, malignes, des Gesichts 256 **

Granulome, tuberkuloide, noch unge-klärter Aetiologie $240 * *$ 
Griseofulvin, therapeutic results in different dermatomycosis and on experimental dermatomycosis in man 148

Griseofulvin, Tinea Imbricata Treated with 158

Griseofulvin, zur Wirkung von auf die experimentelle Trichophytie des Meer-schweinchens 132

Ground Substance $364 * *$

Hoffmann, Erich, in memoriam 129 Homogreffe, reaction cutanée de type,

chez Thomme 264 Hormones $366 * *$

Immunology and Allergy, Current Problems in $259 * * *$

Keratoacanthoma multiplex 239 Keratosis palmo-plantaris mit Periodon-topathie (Papillon-

Lefèvre) 248

384

Register rerum ad vol. 119

Klippel-Trenaunay-Syndrom,Kombina-tion mit Sarkoma Kaposi 45

Kollodiumbabys 164

Kontaktekzem, experimentelles, durch Dinitrochlorbenzol an Ratten und Kaninchen 294

Kveim Antigen, Histopathology of the Reaction Papules Evoked by Intra-dermal Injection of

Normal Tissue Suspensions and 105

Lepra, Histopathologie des Kveim-Tests

105 Lichen nitidus $250 * *$ Lichen ruber planus $126 * *$ Lupus Erythematosus Papularis et Nodularis 79

Makrocheilie, essentielle granulomatöse

$247 * *$ Melanogenesis $364 * *$ Melkersson-Rosenthal'sches Syndrom

$247 * *$ Mowlem et Jackson, etude histologique

de Topération de 264 Mundschleimhaut, Erkrankungen der

$382 * * *$ Mycose expérimentale du cobaye, efifet

de la triamcinolone sur la 75 Mycoses, Traitement des $380 * * *$

Mykose, experimentelledesMeerschwein-

chens, zur Wirkung von Griseofulvin

auf die 132 Mykosen beim Menschen $196 * * *$

Necrobiosis lipoidica $252 * *$ Neurodermitits-Prurigo $123 * *$ Nickelekzem 40

Nickelekzem, Beitrag zur Klinik des 197 Niederländische Dermatologen-Vereini-

gung, 153. Tagung am 30. November

1958 in Utrecht 57

Paget, maladie de, considerations patho-

géniques à $\Gamma$ occasion de $\Gamma$ étude d'un

cas à localisation extra-mammaire 93 Palmoplantarkeratosen, zur Klinik und

Genetik der hereditären papulösen 309 Panniculitis $254 * *$ Paronychia, Chronic, Pathogenesis

and

Treatment of 229 Pautrier, Lucien Marie, Necrologia 378 Periarteriitis nodosa und Gefäßerkrankungen, Bewertung der Riesenzellen

bei der 1 Periodontopathie, Keratosis palmo-plan-

taris mit 248 Pharmacology and Physiology of the

Skin 1958/59 $360 * *$ Phlébologie, Congrès International de,

6,7 et 8 mai 1960 à Chambéry (Savoie,

France) 196 
Photobiologie, Comité International de, Third International Congress in Copenhagen, July 31 to August 5, 1960382

Photobiology $369 * *$

Physiology and Pharmacology of the Skin 1958/59 $360 * *$

Pilze, parasitische beim Menschen $196 * * *$

Prurigo nodularis $125 * *$

Pruritus $121 * *$

Psoriasis-Behandlung, Disulfirampsy-chose bei der 238

Psoriasis vulgaris, über die Pathogenese und zeitgemäße Therapie der 30

Roentgenbestrahlung von Carcinomen, Veränderungen der Haut bei 221

Sarcoid Darier-Roussy's 300

Sarcoma idiopathicum varicosum et

osteohypertrophicum 45 Sarkoma Kaposi, Kombination mit dem

Syndrom von «Klippel-Trenaunay»

45 Scleroderma with Bullous Lesions 341 Sebaceous and Sweat glands 367 ** Sebum, secretion, etude de sujets sains,

épileptiques et parkinsoniens 232 Sensitivity Reactions to Drugs 259 *** Sklerodermie,

umschriebene, über die

für die Lokalisation verantwortlichen

Faktoren bei der 115 Spermatozoen, menschliche, elektronen-

optische Untersuchungen 332 Sneddon-Wilkinson, subcorneal pustular

dermatosis 223 Sulfydryles, groupes, titrage ampéro-

métrique dans la peau brulée 84

Talgsekretion bei Gesunden, Epilepti-kern und Parkinson-Kranken 232

Tinea Imbricata Treated with Griseofulvin 158

Transplantate, homologe, beim Menschen 265

Triamcinolone, efifet de la, sur la mycose expérimentale du cobaye 75

Trichophytie, experimenteUe des Meer-schweinchens, zur Wirkung von Griseofulvin auf die 132 Vascular and Circulation Studies $367 * *$ Venerologie und Dermatologie, Bd. III/1

$260 * * *$ Venerologie und Dermatologie, Bd. III/2 381

Xanthomes-Lentigos disséminés juveniles avec diabète insipide et hyper-lipidémie 328 